\title{
Spin-Polarized Carrier Injection in MOCVD-Grown YBCO/STO/LSMO Heterostructures with Underlying YBCO Layer
}

\author{
B. Vengalis ${ }^{a, *}$, V. Plaušinaitiene ${ }^{a, b}$, A. Abrutis ${ }^{b}$ \\ AND Z. ŠALTYTE $\dot{E}^{b}$ \\ ${ }^{a}$ Semiconductor Physics Institute, A. Goštauto 11, 2600 Vilnius, Lithuania \\ ${ }^{b}$ Vilnius University, Dept. of General and Inorganic Chemistry \\ Naugarduko 24, 2006, Vilnius, Lithuania \\ The oxide heterostructures composed of superconducting $\mathrm{YBa}_{2} \mathrm{Cu}_{3} \mathrm{O}_{7}$ \\ bottom layer, the overlying ferromagnetic $\mathrm{La}_{1-x} \mathrm{Sr}_{x} \mathrm{MnO}_{3}$ film and $\mathrm{SrTiO}_{3}$ \\ as ultrathin $(d \approx 5 \mathrm{~nm})$ barrier were grown heteroepitaxially onto $\mathrm{LaAlO}_{3}$ \\ substrates by applying pulsed liquid injection metalorganic chemical vapour \\ deposition technique. We report anomalous interface resistance increase with \\ cooling just below superconductive transition temperature $\left(T_{\mathrm{c}} \cong 85 \mathrm{~K}\right)$ and \\ enhanced suppression of supercurrent of strip-like $\mathrm{YBa}_{2} \mathrm{Cu}_{3} \mathrm{O}_{7}$ film due to \\ spin-polarized carriers injected from the ferromagnetic manganite layer.
}

PACS numbers: 74.50.+r, 81.15.Gh, 85.25.-j

\section{Introduction}

During the last few years, increasing attention was attributed to metal oxide heterostructures composed of high- $T_{\mathrm{c}}$ superconductors (HTS) and to colossal magnetoresistance (CMR) manganites exhibiting spin-polarized carriers in their ferromagnetic (FM) state. The HTS/I/CMR heterostructures (where I is an ultrathin insulating barrier) are very interesting for research of nonequilibrium superconductivity and offer promising possibility for novel superconducting switching devices based on spin-polarized carrier injection [1-6].

The $\mathrm{YBa}_{2} \mathrm{Cu}_{3} \mathrm{O}_{7}$ (YBCO) films with significantly reduced surface roughness have been grown in this work by an innovative pulsed liquid injection metalorganic chemical vapour deposition (MOCVD) technique. In contrast to earlier works, the prepared HTS/CMR heterostructures containing YBCO bottom layer allowed to

*corresponding author; e-mail: veng@pfi.lt 
inject spin-polarized carriers uniformly into a wide area of superconducting stripe and to reduce possible effect of the Joule heating on critical current of the YBCO superconductor.

\section{Preparation and characterization of the films and heterostructures}

The $\mathrm{YBa}_{2} \mathrm{Cu}_{3} \mathrm{O}_{7} / \mathrm{SrTiO}_{3} / \mathrm{La}_{1-x} \mathrm{Sr}_{x} \mathrm{MnO}_{3}$ (YBCO/STO/LSMO) heterostructures with YBCO as a bottom layer $(d \approx 150 \mathrm{~nm})$ and $\mathrm{SrTiO}_{3}(\mathrm{STO})$ as a barrier $\left(d \approx 5 \mathrm{~nm}\right.$ ) were prepared onto $\mathrm{LaAlO}_{3}(\mathrm{LAO})$ substrates by an innovative pulsed liquid injection MOCVD technique. Y, Ba, Cu, Sr, La, Mn thd complexes (thd - 2,2,6,6-tetramethylheptane-3,5-dionate) and $\mathrm{Ti}(\mathrm{i}-\mathrm{OPr})_{2}(\text { thd })_{2}$ were used as the precursor materials and monoglyme (1,2-dimethoxyethane) as a solvent. Deposition at $820^{\circ} \mathrm{C}$ under optimized vapour phase composition allowed to grow single phase epitaxial YBCO films with significantly improved surface quality.

Fabrication of the heterostructures consisted of several technological procedures. Ti coatings were firstly deposited onto masked $\mathrm{LaAlO}_{3}$ substrates by means of DC magnetron sputtering. In following, a stripe-like superconducting YBCO film (0.5 mm wide and $5.0 \mathrm{~mm}$ long) was grown in situ at $820^{\circ} \mathrm{C}$ onto uncoated parts of the substrate since the YBCO film deposited on $\mathrm{Ti}\left(\mathrm{TiO}_{2}\right)$-coated substrate was found to be highly resistive. Ultrathin STO barrier $(d \approx 5 \mathrm{~nm})$ and the LSMO top layer were deposited subsequently onto the HTS film. Finally, Ag coatings were sputtered onto the top LSMO layer to fabricate low resistance contacts for electrical measurements.

Resistance versus temperature, critical current $J_{\mathrm{c}}$ and voltage versus current were measured for the patterned stripe-like YBCO films. The interface resistance between HTS and manganite layer was measured by applying three-probe technique and passing current perpendicular to the film plane, i.e. between underlying HTS film and low resistance Ag electrode deposited onto the LSMO layer.

\section{Results and discussion}

X-ray diffraction (XRD) and reflection high-energy electron diffraction (RHEED) investigations revealed epitaxial quality of the prepared films and heterostructures. The corresponding values of roughness average $\left(R_{\mathrm{a}}\right)$ of about $1.5 \mathrm{~nm}$ and $\approx 1.4 \mathrm{~nm}$ have been estimated for the YBCO/LAO $(d \approx 150 \mathrm{~nm})$ and LSMO/LAO $(d \approx 130 \mathrm{~nm})$ films, respectively.

Figure 1a demonstrates temperature-dependent effective resistance, $R=$ $U_{34} / I_{15}$ of the YBCO stripe for the prepared YBCO/STO/LSMO heterostructure (here $I_{15}$ is electrical current flowing between points 1 and 5 and $U_{34}$ is the corresponding voltage measured between points 3 and 4 as seen from the inset to 

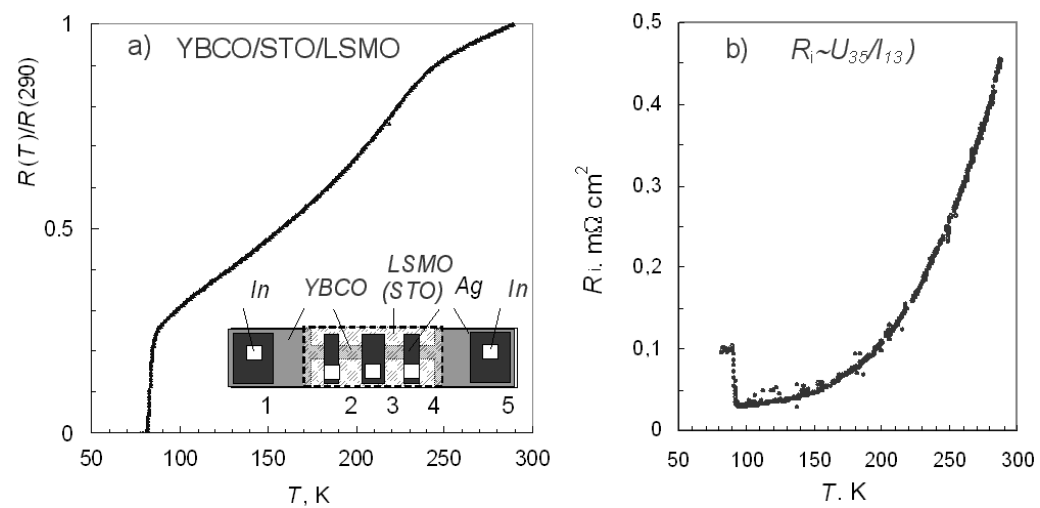

Fig. 1. (a,b) Effective resistance of YBCO stripe $\left(R=U_{24} / I_{15}\right)$ measured for the YBCO/STO/LSMO heterostructure (a) and resistivity of the interface $\left(R_{\mathrm{i}} \sim U_{35} / I_{13}\right)$ formed between HTS and manganite layer (b).

Fig. 1a). Resistance anomaly seen in Fig. 1a at $T=220-250 \mathrm{~K}$ is due to the characteristic paramagnetic-ferromagnetic transition of the overlaying manganite film while sharp resistance drop at 85-87 K demonstrates superconducting transition of the underlaying YBCO film. The interface resistance between HTS and manganite layers $\left(R_{\mathrm{i}}=U_{35} S / I_{13}\right.$, here $S$ is the interface square) was measured separately by applying three-probe method, i.e. by passing current between HTS film and low resistance Ag electrode deposited onto the LSMO film (points 1 and 3, respectively) and measuring voltage drop between points 3 and 5 . It is important to note relatively low interface resistivity values $\left(R_{\mathrm{i}} \cong 2 \times 10^{-5} \Omega \mathrm{cm}^{2}\right.$ at $\left.T=100 \mathrm{~K}\right)$ and anomalous $R_{\mathrm{i}}$ increase with cooling just below $T_{\mathrm{c}}$. This effect demonstrates a depressed Andreev reflection of spin-polarized carriers injected into the superconducting YBCO film from ferromagnetic manganite layer. The unusual voltage $\left(U_{35}\right)$ versus current $\left(I_{13}\right)$ curves of the same interface demonstrating step-like behaviour at certain current values (see Fig. 2) may be understood assuming interface resistance decrease due to current-induced superconducting-normal state $(\mathrm{S}-\mathrm{N})$ transition occurring in the superconducting film.

Figure 3 shows typical voltage versus current of the patterned superconducting YBCO film of the YBCO/STO/LSMO heterostructure. Nonzero resistance of the heterostructure (at $T<T_{\mathrm{c}}$ and low current values) seen in the figure may be explained assuming shunting of the YBCO film resistance by highly resistive $\left(>10^{5} \Omega\right)$ films of polycrystalline YBCO and LSMO deposited onto Ti-coated parts of the heterostructure. In such a case, critical current of the YBCO stripe has been estimated from the observed onset of the nonlinearity in the measured $U-I$ curves. Our estimations gave $J_{\mathrm{c}}$ values of about $2.0 \times 10^{5} \mathrm{~A} / \mathrm{cm}^{2}$ at $78 \mathrm{~K}$ for the YBCO stripes. In most cases, the $I_{\mathrm{c}}$ values for spin-polarized carriers injected from the LSMO layer $\left(I_{34}\right.$ and $\left.U_{15}\right)$ were lower compared to those corresponding to nonpolarized electrons $\left(I_{15}\right.$ and $\left.U_{34}\right)$. Relatively low current gain 

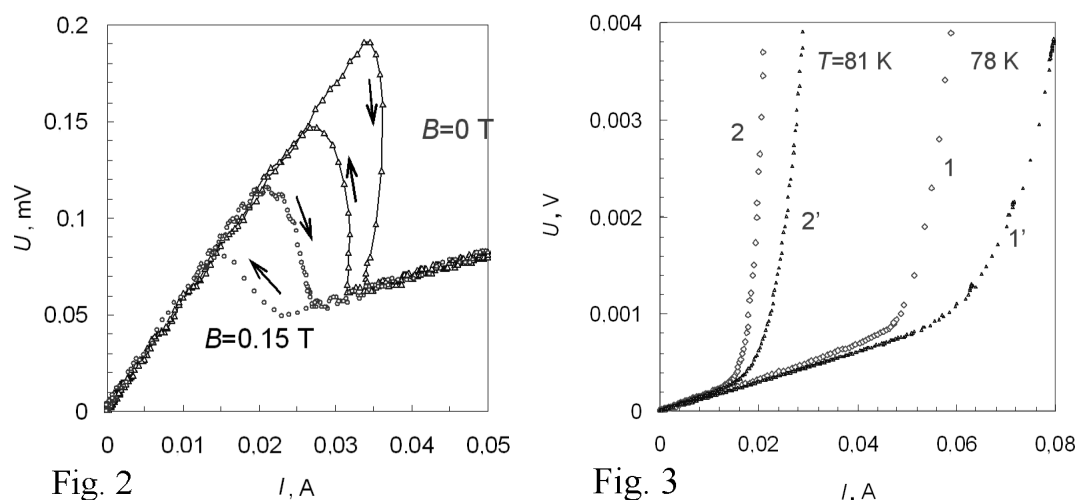

Fig. 3

I, A

Fig. 2. Voltage-current $\left(U_{35}-I_{13}\right)$ dependencies of the YBCO/LSMO interface measured by three probe method during heating and cooling at $B=0 \mathrm{~T}$ and $1.2 \mathrm{~T}$.

Fig. 3. Voltage versus current measured for the YBCO/STO/LSMO heterostructure at $T=78 \mathrm{~K}\left(1,1^{\prime}\right)$ and $82 \mathrm{~K}\left(2,2^{\prime}\right)$ by injecting spin polarized $\left(I_{24}, U_{12}\right)-(1,2)$ and nonpolarized $\left(I_{12}, U_{24}\right)-\left(1^{\prime}, 2^{\prime}\right)$ carriers.

$\left(G=\Delta I_{\mathrm{sp}} / \Delta I_{\mathrm{np}} \leq 1.3\right)$ estimated in this work for the YBCO/STO/LSMO heterostructures may, probably, be understood assuming presence of intermediate layer with depressed FM properties at the YBCO/LSMO interface. Both technological processes and investigations of the structures are in progress in order to enhance the role of spin-polarized carrier injection on critical parameters of the YBCO films.

\section{Acknowledgments}

This work was partially supported by CE project PRAMA.

\section{References}

[1] Z.W. Dong, R. Remesh, T. Venkatesan, M. Johnson, Z.Y. Chen, S.P. Pai, V. Talyansky, R.P. Sharma, R. Shrekala, C.J. Lob, Appl. Phys. Lett. 71, 1718 (1997).

[2] N.-C. Yeh, R.P. Vasquez, C.C. Fu, A.V. Samoilov, Y. Li, K. Vakii, Phys. Rev. B 60, 10522 (1999).

[3] A.M. Goldman, P.I. Kraus, K. Nikolaev, V. Vasko, A. Bhattacharya, W. Cooley, J. Superconduct. 14, 283 (2001).

[4] V. Plausinaitiene, A. Abrutis, B. Vengalis, R. Butkute, J.P. Sénateur, Z. Saltyte, V. Kubilius, Physica C 351, 13 (2001).

[5] Y. Gim, A.W. Kleinsasser, J.B. Barner, J. Appl. Phys. 90, 4063 (2001).

[6] B. Vengalis, V. Plaušinaitienè, A. Abrutis, R. Butkutè, J.P. Sénateur, Z. Šaltytė, J. Phys. (France) IV 11, 53 (2001). 